\title{
Semelhança fraterna nos níveis de aptidão física: um estudo na população rural de Calanga, Moçambique
}

CDD. 20.ed. 613.7

614.49

\author{
Silvio SARANGA \\ Leonardo NHANTUMBO* \\ Rogério César FERMINO** \\ António PRISTA* \\ André SEABRA*** \\ José António Ribeiro MAIA***
}

${ }^{*}$ Faculdade de

Ciências de Educação

Física e Desporto,

Universidade

Pedagógica -

Moçambique.

**Universidade Federal

do Paraná.

***Faculdade de Des-

porto, Universidade do

Porto - Portugal.

\section{Resumo}

Este estudo teve dois propósitos: 1) determinar agregação familiar em diferentes indicadores da aptidão física, e 2) a sua componente genética $\left(h^{2}\right)$. A amostra foi constituída por 330 sujeitos (174 meninos e 156 meninas) dos sete aos 17 anos de idade, pertencentes a 132 famílias de Calanga, uma localidade rural de Moçambique. A aptidão física foi avaliada com os seguintes testes: sentar e alcançar, impulsão horizontal, tempo de suspensão na barra, dinamometria manual, corrida da milha, abdominais e corrida vaivém (10 × $5 \mathrm{~m}$ ). Para estimar semelhança entre irmãos foram calculadas correlações e a sua contribuição genética foi realizada pelo método da máxima verosimilhança para estimar componentes de variância. Todos os cálculos foram ajustados para a idade, sexo, idade ${ }^{2}$, idade ${ }^{3}$, idade*sexo, idade ${ }^{2 *}$ sexo, bem como um "score" de atividade física. A análise de dados foi realizada no programa de Epidemiologia Genética SAGE. As correlações foram baixas em cada indicador de aptidão física: irmãos (entre 0,01 e 0,25), irmãs (entre -0,19 e 0,45) irmão-irmã (entre -0,02 e 0,39). A componente genética em cada item de aptidão foi a seguinte: sentar e alcançar $h^{2}=0,41 \pm 0,16, p=0,01$; impulsão horizontal $h^{2}=0,60 \pm 0,14, p=0,001$; tempo de suspensão na barra $h^{2}=0,18 \pm 0,20, p=0,37$; dinamometria manual $h^{2}=0,19 \pm 0,13$, corrida da milha $h^{2}=0,49 \pm 0,14, p=0,001$; abdominais $h^{2}=0,09 \pm 0,13, p=0,59 ; p=0,16$; corrida vaivém $h^{2}=0,07 \pm 0,15, p=0,63$. Conclusões: 1) a agregação familiar foi mais elevada nas irmãs do que nos irmãos do mesmo sexo e sexo oposto; 2) foram encontrados fatores genéticos moderados na flexibilidade, capacidade aeróbia e força explosiva; 3 ) a variância entre irmãos nos outros fenótipos foi explicada por fatores de natureza ambiental que podem estar relacionados com atividades de subsistência familiar.

Unitermos: Epidemiologia genética; Fratrias; Aptidão física; Moçambique.

\section{Introdução}

O estudo das mais variadas características (i.e. fenótipos) do "Homo Sapiens" continua a merecer um forte fascínio em antropólogos físicos e especialistas em Biologia Humana. Essencialmente, procuram entender aspectos da história natural das alterações morfofuncionais ao longo do tempo em resposta às mais diversas influências ambientais. Uma das características mais interessantes de estudo é a que se refere à aptidão funcional e à enorme variabilidade da sua expressão em termos populacionais que, em certa medida, reflete diferenças interindividuais nos níveis de atividade física ao longo da história recente do homem, i.e., desde as exigências sentidas quando caçadorcoletor às que são emergentes na atualidade (BROWN \& Konner, 1987; Cordain, Gotshall, Eaton \& Eaton, 1998). Decorre daqui, de forma clara, que o passado histórico e biológico do "Homo Sapiens" foi expresso por um cotidiano rico em atividades físicas de índole diversificado manifestado pela sua elevada eficiência em termos de sobrevivência marcada pela sua forte interação com o seu envolvimento físico (SHEPHARD, 1997). Atualmente, tal como no 
passado, nos países em transição sociodemográfica e nutricional, fundamentalmente nos contextos rurais, as atividades de sobrevivência e as exigências ao nível da aptidão física ou funcional requeridas para as tarefas do cotidiano são bem mais complexas e exigentes do que as que se observam nos países altamente industrializados dominados por uma tecnologia sofisticada. Nos países em transição, como é o caso da maioria dos países do continente africano, a eficiência em realizar trabalho contínuo é determinante, o que torna a capacidade funcional de qualquer indivíduo, ou grupo populacional, um elemento essencial para a sua capacidade produtiva de sobrevivência local (PRISTA, MARQUES \& MAIA, 1997).

É assim que as preocupações em torno do estudo da aptidão física neste continente, ainda que diferentes das preocupações dos investigadores dos países industrializados, são igualmente relevantes face à novidade da crescente urbanização das sociedades africanas. Um dos seus efeitos imediatos parece ter sido o aumento inesperado da prevalência de inatividade física nunca até então alcançado em termos populacionais (BROWNSON, BOEHMER \& LUKE, 2005). Esta é uma preocupação recente em termos de Saúde Pública. A pesquisa no âmbito da aptidão física nestes países não é tão vasta quanto se desejaria. A maior parte procura contrastar níveis de aptidão física das populaçôes dos espaços urbanos e rurais, e circunscreve-se a países como a África do Sul (Henneberg, Brush $\&$ Harrison, 2001), Botswana (Corlet, 1988), Egito (ELNaShIR \& MaYHEW, 1984), Moçambique (Muria, Prista \& Maia, 2000; Prista, 1995; Prista, Marques \& Maia, 1997), Senegal (Faye, Seck \& Cisse, 1999) e Zaire (Guesquière, D’Hulst \& Nkiama, 1989; Nkiama, 1993). A aptidão física, enquanto característica multidimensional de cada indivíduo, expressa em termos populacionais uma enorme variabilidade que se pensa estar dependente de fatores genéticos e ambientais (Bouchard, Malina \& Pérusse, 1997). A informação acerca dos efeitos genéticos a governar os níveis distintos de aptidão física analisados com base em baterias de testes de validade reconhecida não é muito extensa (KatzmarzyK, Gledhill, Pérusse $\&$ BOUCHARD, 2001). Os estudos que possuem informação sobre famílias nucleares e irmandades não gemelares foram realizados no Canadá (BOUCHARD, Daw, Rice, Pérusse, Gagnon, Province, Leon,
RaO, Skinner \& Wilmore, 1998; KatzmarzyK et al., 2001; Pérusse, Leblanc \& Bouchard, 1988; Perusse, Leblanc, Tremblay, Allard, Theriault, Landry, Talbot \& Bouchard, 1987; Pérusse, Lortie, Leblanc, Tremblay, Theriault \& Bouchard, 1987), Estados Unidos (Sallis, Patterson, Morris, Nader \& Buono, 1989) e Portugal (Fernandes \& Maia, 2006; Maia, Lopes \& Morais, 2001; MaiA, Lopes, Seabra \& Garganta, 2003; Vasques, 2005). Por exemplo, PÉrusse et al. (1987a) pesquisaram aspectos de agregação familiar nas componentes da aptidão física, fatores de risco de doenças cardiovasculares e função pulmonar em 304 famílias canadenses, tendo constatado valores de correlação (r) entre 0,21 e 0,34, em parentes de graus distintos. SALLIS et al. (1989) pesquisaram 206 famílias de origem mexicana e anglófona e constataram a presença de uma baixa agregação familiar nos valores da capacidade aeróbia: $0,04 \leq \mathrm{r} \leq 0,35$ nos anglófonos, e $0,03 \leq \mathrm{r}$ $\leq 0,50$ nas famílias mexicanas. No que concerne a pesquisas com fratrias, o único estudo localizado em Portugal foi o de VASQUES (2005), em que procurou averiguar a semelhança na aptidão física em 108 pares de irmãos de ambos os sexos dos 10 aos 18 anos de idade. Os valores de correlação entre irmãos foram baixos a moderados $(-0,08$ a 0,49$)$ nos testes de flexibilidade do tronco, abdominais, flexão de braços e corrida da milha, sendo as meninas mais semelhantes entre si do que os meninos. Pérusse et al. (1987a) em uma amostra canadense constituída por 13.804 indivíduos aparentados, observaram na flexibilidade do tronco, flexão de braços, abdominais e força de preensão, estimavas de heritabilidade $\left(h^{2}\right)$ de 48, 44, 37 e $37 \%$, respectivamente. Tanto quanto pensamos saber, pesquisas sobre a influência dos aspectos genéticos na variabilidade dos níveis de aptidão física das populações de países em transição sociodemográfica, principalmente nos que se localizam na África SubSahariana, são praticamente inexistentes.

Face a esta lacuna informacional, a presente pesquisa têm como principais propósitos: 1) identificar o nível de agregação dos irmãos nas distintas componentes da aptidão física, e 2) determinar a proporção da variabilidade observada em características complexas da aptidão física que é explicada por fatores genéticos e ambientais em irmãos de idade escolar da população rural de Calanga, Moçambique. 


\section{Material e métodos}

Moçambique fica situado na costa oriental do continente africano, tendo um território de $799.390 \mathrm{~km}^{2}$, sendo a sua maior parte constituída por um planalto pouco elevado (FIGURA 1). A população moçambicana foi oficialmente estimada em aproximadamente 20 milhões de habitantes (MoçAMBIQUe. Instituto Nacional de Estatística - INE, 2006). A localidade de Calanga pertence ao Distrito da Manhiça, situa-se a $75 \mathrm{~km}$ a norte de Maputo, tem uma superfície de $2.373 \mathrm{~km}^{2}$, o seu limite ao Norte é o distrito de $\mathrm{Ma}$ gude, a Nordeste o distrito de Bilene, Oeste o Oceano Índico, a Sul o distrito de Marracuene e a Sudeste o distrito da Moamba. A população de Calanga foi estimada em 9.451 pessoas, sendo 3361 crianças na faixa etária compreendida entre os seis e os 20 anos de idade (MoçAMBIQUe. INE, 2006).

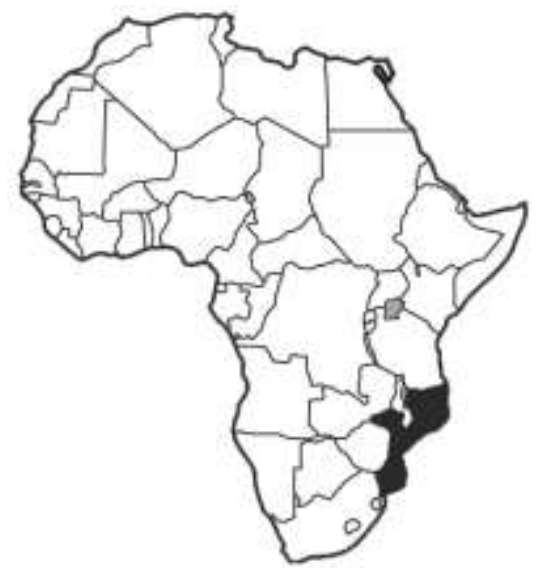

FIGURA 1 - Localização de Moçambique destacada em preto.

A principal fonte de subsistência da população é a agricultura familiar. As famílias desta comunidade são classificadas como pertencentes a um estrato socioeconômico baixo. As habitações são de construção precária, não existe água canalizada nem energia, não há assistência médica e a rede escolar é deficitária. As vias de acesso são de terra batida, não sendo transitáveis em alguns períodos do ano.

Os objetivos do estudo foram antecipadamente explicados aos pais, bem como às direçôes das escolas, chefe e oficiais do posto administrativo e líderes comunitários. Aos encarregados de educação com alguma literacia foi-lhes solicitados para que lessem e assinassem um consentimento informado preparado para o efeito, e que detalhava os objetivos e procedimento essenciais do estudo; os encarregados de educação sem literacia, o mesmo consentimento informado foi-lhes lido em voz alta e explicado em língua local, tendo-lhes sido solicitado a impressão digital do dedo indicador direito como prova do seu consentimento e anuência à pesquisa. $\mathrm{O}$ estudo foi aprovado pelas autoridades nacionais de saúde e de educação de Moçambique e pelo Comitê Nacional de Bioética para a Saúde.

\section{Amostra}

A amostra foi constituída por 330 indivíduos (174 do sexo masculino, 156 do sexo feminino) pertencentes a 132 famílias, cujas irmandades variam entre dois e sete elementos, pertencentes ao escalão etário que se situa entre os sete e os 17 anos de idade cronológica. Os sujeitos constituintes da amostra representam aproximadamente $10 \%$ da população total de Calanga na faixa etária dos seis aos 20 anos de idade. Eram todos estudantes e residiam na localidade de Calanga-Manhiça, província de Maputo, Moçambique. A idade média dos meninos era de 11,5 $\pm 2,6$ anos e das meninas era de 10,7 \pm 3 anos. Estes sujeitos fazem parte da primeira etapa do projeto "Estudo da Variabilidade Biológica em Moçambique”, que tem como principal propósito oferecer informação sobre a variabilidade na população moçambicana, seu significado e alcance na componente da saúde pública, das políticas socioeconômicas e educativas.

\section{Medidas antropométricas e de aptidãofísica}

As medidas antropométricas utilizadas foram a altura e o peso. Os procedimentos técnicos adotados para obtenção destas medidas somáticas foram descritos e padronizados por LoHMAn, Roche e Malina (1988). Para a altura foi utilizado o estadiômetro da marca Harpender com aproximação aos milímetros. $\mathrm{O}$ peso foi medido na balança de marca Secca com aproximação aos $0,5 \mathrm{~kg}$. Os dados da aptidão física foram coletados através da bateria de testes da AAHPERD (1980), que compreendeu provas de flexibilidade (sentar e alcançar), força explosiva dos membros inferiores (impulsão horizontal), força de resistência abdominal (abdominais), resistência cardiorespiratória (corrida da milha), bem como o teste de agilidade (corrida vaivém $10 \times 5 \mathrm{~m}$ ), força de resistência dos membros superiores (tempo de suspensão na barra), dinamometria manual (preensão manual) avaliada por dinamômetro adaptável marca Takei e descritos no programa de testes da bateria do EUROFIT (CoUNCIL OF EUROPE, 1988). Foram permitidas duas repetiçōes a cada sujeito em todas as provas, à exceção na corrida da milha, abdominais e corrida de vaivém. 


\section{Procedimentos estatísticos}

O quadro descritivo das variáveis contou com as medidas básicas, ou seja, a média e o desvio padrão. Estes cálculos foram precedidos de uma análise exploratória com o propósito de verificar eventuais erros de entrada de informação, a presença de "outliers" e inspecionar a normalidade das distribuições através do teste de Kolmogorov-Smirnov. Nos testes de tempo de suspensão na barra, abdominais e preensão manual identificaram-se problemas de normalidade na sua distribuição, pelo que se optou pela transformação logarítmica dos seus valores. Seguidamente, foi utilizado o "software" Pedstats (WigGinTon \& ABECASIS, 2005) para verificar eventuais erros na estrutura de cada fratria. Neste "software" foi também efetuada a análise exploratória da informação no que diz respeito aos indicadores da aptidão física no seio de cada fratria.
$\mathrm{Na}$ segunda etapa, com o propósito de remover o efeito de covariáveis dos fenótipos em estudo recorreuse à regressão linear múltipla com solução "stepwise". As covariáveis testadas foram as seguintes: altura, peso, idade, sexo, idade ${ }^{2}$, idade $^{3}$, sexo*idade, sexo*idade ${ }^{2}$ e coeficiente de atividade física. Este coeficiente foi sugerido por PRISTA, MARQUeS e MAIA (2000) para estimar um compósito linear que reflete o dispêndio energético diário. Do melhor modelo de regressão em cada um dos fenótipos testados, foram considerados os resíduos estandardizados para cálculo posterior das correlações intra familiares para estimar $a h^{2}$. Uma parte substancial dos cálculos foi realizada no "software" estatístico SPSS 15.0; a h² (módulo ASSOC) e as correlaçôes entre irmandades (módulo FCOR) foram estimadas no "software" de Epidemiologia Genética SAGE (CASE WeSteRn Reserve UnIVERSITY, 2005). Em todas as análises foi estabelecido um nível de significância de 5\%.

\section{Resultados}

A TABELA 1 apresenta os resultados das estatísticas descritivas das diferentes componentes da aptidão física. Os irmãos apresentam desempenhos médios mais elevados em todas as provas, à exceção da flexibilidade. É importante salientar a grande variabilidade expressa pela amplitude dos resultados nos dois sexos, não obstante a similaridade da variância dos valores em cada componente da aptidão física.

TABELA 1 - Valores médios e desvio-padrão das sete componentes da aptidão física das diversas fratrias.

\begin{tabular}{lcccc}
\hline \multirow{2}{*}{ Testes de aptidão física } & \multicolumn{4}{c}{ Fratrias } \\
\cline { 2 - 5 } & \multicolumn{2}{c}{ Irmãos $(\mathbf{n}=\mathbf{1 7 3})$} & \multicolumn{2}{c}{ Irmãs $(\mathbf{n}=\mathbf{1 5 5})$} \\
\cline { 2 - 5 } & $\mathbf{m} \pm \mathbf{d p}$ & $\mathbf{a m p l i t u d e}$ & $\mathbf{m} \pm \mathbf{d p}$ & amplitude \\
\hline Idade (anos) & $11,5 \pm 2,6$ & $7-17$ & $10,7 \pm 3$ & $7-17$ \\
Sentar e alcançar $(\mathrm{cm})$ & $34,9 \pm 6,3$ & $17-50$ & $36,9 \pm 6,5$ & $19-50$ \\
Impulsão horizontal $(\mathrm{cm})$ & $144 \pm 29,2$ & $79,2-217$ & $133,7 \pm 30,9$ & $50-194$ \\
Tempo de suspensão na barra $(\mathrm{s})$ & $10,3 \pm 6,1$ & $2-26$ & $9,4 \pm 6$ & $2-25$ \\
Abdominais (no rep) & $7,6 \pm 5,5$ & $1-22$ & $6,6 \pm 5,7$ & $1-25$ \\
Dinamometria manual $(\mathrm{kg} / \mathrm{f})$ & $19,7 \pm 8,9$ & $6,7-47$ & $15,6 \pm 7,3$ & $6,3-35$ \\
Corrida vaivém $10 \times$ x $5 \mathrm{~m}(\mathrm{~s})$ & $23,6 \pm 2,1$ & $20-30$ & $24,5 \pm 2,1$ & $20-30$ \\
Corrida da milha $(\mathrm{s})$ & $491,9 \pm 67,6$ & $244-646$ & $551,3 \pm 93,9$ & $362-845$ \\
\hline
\end{tabular}

A TABELA 2 contém informação relativa às estimativas de $\mathrm{h}^{2}$, sendo elevada no teste de impulsão horizontal $(\approx 60 \%)$, moderada na corrida da milha $(\approx$ $49 \%)$ e no teste de sentar e alcançar $(\approx 41 \%)$, baixa no tempo de suspensão na barra $(\approx 18 \%)$ e força de preensão manual $(\approx 19 \%)$, e não diferente de zero no teste de abdominais e corrida vaivém. Estes resultados parecem sugerir influências genéticas e ambientais distintas a governar a variabilidade nos vários fenótipos da aptidão funcional entre irmãos neste grupo populacional. 
TABELA 2 - Valores de estimativa de heritabilidade $\left(h^{2}\right), \pm$ erro padrão e valor de p dos indicadores da aptidão física

\begin{tabular}{|c|c|c|c|c|c|}
\hline \multirow{2}{*}{$\begin{array}{c}\text { Indicadores } \\
\text { da aptidáo física }\end{array}$} & \multicolumn{3}{|c|}{ Componentes de variância } & \multirow{2}{*}{$\mathbf{h}^{2}$} & \multirow{2}{*}{$\mathbf{p}$} \\
\hline & Poligênica & Residual & Variância total & & \\
\hline Sentar e alcançar & $0,38 \pm 0,16$ & $0,57 \pm 0,14$ & $0,95 \pm 0,08$ & $0,41 \pm 0,16$ & 0,010 \\
\hline Impulsão horizontal & $0,59 \pm 0,16$ & $0,39 \pm 0,13$ & $0,99 \pm 0,09$ & $0,60 \pm 0,14$ & 0,001 \\
\hline Tempo de suspensão na barra & $0,17 \pm 0,19$ & $0,77 \pm 0,19$ & $0,94 \pm 0,09$ & $0,18 \pm 0,20$ & 0,372 \\
\hline Abdominais & $0,02 \pm 0,03$ & $0,16 \pm 0,04$ & $0,17 \pm 0,02$ & $0,1 \pm 0,19$ & 0,596 \\
\hline Dinamometria manual & $0,17 \pm 0,12$ & $0,73 \pm 0,13$ & $0,89 \pm 0,07$ & $0,19 \pm 0,13$ & 0,160 \\
\hline Corrida vaivém $(10$ x 5) & $0,07 \pm 0,15$ & $0,93 \pm 0,17$ & $1 \pm 0,09$ & $0,07 \pm 0,15$ & 0,632 \\
\hline Corrida da milha & $0,45 \pm 0,15$ & $0,47 \pm 0,13$ & $0,92 \pm 0,09$ & $0,49 \pm 0,14$ & 0,001 \\
\hline
\end{tabular}

As correlações entre fratrias nas sete componentes da aptidão física são apresentadas na TABELA 3. As correlações são baixas $(-0,01 \leq \mathrm{r} \leq 0,23)$ entre irmãos, com excepção da prova de suspensão na barra e corrida da milha em que se revelaram "moderadas" com os valores de $\mathrm{r}$ a oscilarem entre 0,25 e 0,31 . Nas irmãs, os resultados são baixos nas provas de suspensão na barra, abdominais, corrida vaivém e preensão manual $(-0,19 \leq \mathrm{r}$ $\leq 0,19)$ e moderadas no teste de sentar e alcançar, impulsão horizontal e na corrida da milha $(0,35 \leq \mathrm{r} \leq 0,45)$. Nos irmãos de sexo oposto, à exceção das provas de sentar e alcançar e impulsão horizontal com valores moderados de 0,25 e 0,39 , nos outros indicadores da aptidão física os resultados $r$ são muito baixos, entre -0,02 e 0,18.

TABELA 3 - Correlações ( \pm erro-padrão) dos indicadores da aptidão física das diversas fratrias.

\begin{tabular}{lccc}
\hline & \multicolumn{3}{c}{ Fratrias } \\
\cline { 2 - 4 } \multicolumn{1}{c}{ Testes de aptidão física } & $\begin{array}{c}\text { Irmãos } \\
\text { (pares }=\mathbf{7 0})\end{array}$ & $\begin{array}{c}\text { Irmãs } \\
(\text { pares }=\mathbf{6 6})\end{array}$ & $\begin{array}{c}\text { Irmãos do sexo oposto } \\
\text { (pares = 134) }\end{array}$ \\
\cline { 2 - 4 } & $\mathbf{r} \pm \mathbf{~ e p}$ & $\mathbf{r} \pm \mathbf{~ e p}$ & $\mathbf{r} \pm$ ep \\
\hline Sentar e alcançar & $0,01 \pm 0,13$ & $0,35 \pm 0,14$ & $0,25 \pm 0,10$ \\
Impulsão horizontal & $0,23 \pm 0,13$ & $0,44 \pm 0,14$ & $0,39 \pm 0,09$ \\
Tempo de suspensão na barra & $0,31 \pm 0,17$ & $-0,19 \pm 0,14$ & $0,09 \pm 0,11$ \\
Abdominais & $0,1 \pm 0,18$ & $0,03 \pm 0,18$ & $-0,02 \pm 0,14$ \\
Dinamometria manual & $0,09 \pm 0,13$ & $0,19 \pm 0,14$ & $0,02 \pm 0,1$ \\
Corrida vaivém (10 x 5 m) & $0,13 \pm 0,15$ & $0,04 \pm 0,15$ & $-0,02 \pm 0,10$ \\
Corrida/caminhada da milha & $0,25 \pm 0,16$ & $0,45 \pm 0,15$ & $0,18 \pm 0,12$ \\
\hline
\end{tabular}

\section{Discussão}

É evidente que fatores genéticos e do envolvimento, bem como a sua interação e covariação, são agentes de forte responsabilidade na explicação da variação nos níveis da aptidão física a nível populacional. A importância dos fatores genéticos nas componentes da aptidão física tem sido estudada em famílias nucleares (Bouchard, AN, Rice, SKINner, Wilmore, Gagnon, Pérusse, Leon \& Rao, 1999; Bouchard et al., 1998; KaTZMARZYK et al., 2001; Pérusse, Gagnon, Province, Rao, Wilmore,
Leon, Bouchard \& Skinner, 2001; Pérusse, Lortie, Leblanc, Tremblay, Theriault \& Bouchard, 1987b; Pérusse et al., 1987a) gêmeos (BEUnen \& Thomis, 2000; Beunen, Thomis, Peeters, Maes, Claessens \& Vlietinck, 2003; Fernandes \& Maia, 2006; Maia, Lopes \& Morais, 2001; Maia et al., 2003; Silberg, Meyer, Maes, Hewitt, Simonoff, PiCKLes, Loeber \& EAVEs, 1996) e fratrias (VASQues, 2005) de populações de países desenvolvidos. No entanto, existe uma escassez de estudos sobre a 
influência desses fatores na variabilidade fenotípica das componentes da aptidão física em populações de países em transição sociodemogáfica, o que limita a comparação dos resultados do presente estudo com os que estão disponíveis na literatuta conduzindo, necessariamente, a alguma insuficiência explicativa.

Os valores médios na amostra de Calanga dos distintos fenótipos da aptidão física mostram diferenças entre irmãos e irmãs, a favor dos irmãos, com exceção do teste de sentar e alcançar em que as irmãs apresentam melhor performance. Quando comparados com os valores médios da cidade de Maputo (PRISTA, MarQUes \& Maia, 1997; Saranga, Prista \& Maia, 2002), possuem desempenhos superiores nos testes de sentar e alcançar, preensão manual, corrida vaivém e corrida da milha. Os valores médios da componente capacidade aeróbia na amostra de Calanga são superiores aos dos americanos das mesmas idades e sexo (AAHPERD, 1980); na flexibilidade, tempo de suspensão na barra e corrida vaivém possuem desempenhos motores mais elevados que os belgas (Council of Europe, 1988, 1993), mas não nas provas de impulsão horizontal e força de preensão manual.

A interpretação da agregação interfratrias nas componentes da aptidão física da população rural de Calanga baseou-se na estimativa de $\mathrm{h}^{2}$. As pesquisas em famílias nucleares (Bouchard, MaLina \& Pérusse, 1997; Bouchard et al., 1998, 1999; PÉRUSSe et al., 1987a, 1987b, 2001), e em gêmeos (Beunen et al., 2003; Fernandes \& Maia, 2006), têm mostrando que uma fatia importante da variação nas características complexas da aptidão física é hereditária. A magnitude deste efeito pode ser condicionada por diferentes variáveis confundidoras em sujeitos aparentados vivendo em países em desenvolvimento.

Os resultados da presente pesquisa salientam a influência dos fatores genéticos na variação interfratrias nos indicadores da aptidão física, com valores de $h^{2}$ entre $0,41 \leq h^{2} \leq 0,60$ para o teste de sentar e alcançar, corrida da milha e impulsão horizontal. Contrariamente, para o teste de vaivém, abdominais, tempo de suspensão na barra e preensão manual, os resultados parecem não evidenciar qualquer dependência genética, com estimativas de $h^{2}$ a salientarem-se entre $0,07 \leq h^{2} \leq 0,19$. Tais resultados sugerem uma forte influência do envolvimento não comumente partilhado, associados a fatores de natureza cultural própria das famílias.

No teste de sentar e alcançar, que avalia a componente da flexibilidade dos músculos ísquiotibiais, o valor de $\mathrm{h}^{2}$ foi de aproximadamente $41 \%$. Os valores do desempenho na flexibilidade são bem específicos de cada articulação, estando normalmente relacionada com a morfologia, a idade, sexo e nível de atividade física (Bouchard, Malina \& Pérusse, 1997). As estimativas relativamente elevadas observadas neste fenótipo podem ser parcialmente explicadas pela interferência de aspectos morfológicos da articulação do quadril (Bouchard, Malina \& Pérusse, 1997) e dos músculos ísquiotibiais (KATZMARZYK et al., 2001). Acresce o fato da amostra ser de um meio rural, com atividades diárias de subsistência intensas que solicitam fortemente a musculatura dorso-lombar. A informação disponível sobre a influência genética na variabilidade deste fenótipo não é tão extensa quanto o desejado. Dados disponíveis referem valores moderados a elevados de $\mathrm{h}^{2}$ (entre 18 e 69\%) para diferentes manifestações de flexibilidade de articulações distintas avaliadas em condiçôes diversas (BOUCHARD, MaLINA \& Pérusse, 1997; KatzmarzyK et al., 2001). Esta dispersão de resultados é devida, sobretudo, a diferenças na amostragem, tipo de indicadores utilizados para marcar a flexibilidade e o processo de avaliação.

$\mathrm{Na}$ prova de impulsão horizontal, que avalia a força explosiva dos membros inferiores, a estimativa $\mathrm{de}^{2}$ foi de $60 \%$. Este valor está no mesmo sentido dos estudos revistos, que apontam para uma forte influência genética nas várias formas de expressar força muscular. Habitualmente o valor de $\mathrm{h}^{2}$ varia entre 27 e 74\% em famílias (BEUNEN et al., 2003; BOUCHARD, Malina \& Pérusse, 1997; KatzmarzyK et al., 2001) e entre 14 e $85 \%$ em gêmeos (Beunen \& ThOMIs, 2000; BEUNEN et al., 2003). Isto quer dizer que uma parte substancial da variação neste fenótipo é devida aos fatores genéticos. No entanto, importa referir que os valores de $\mathrm{h}^{2}$ reportados na literatura variam em função do tipo de força avaliada (estática, dinâmica ou explosiva) e do teste utilizado (Malina, 1986). Todavia, nos testes de abdominais, tempo de suspensão na barra e preensão manual, que avaliam a forçal resistência abdominal, força dos membros superiores e a força de preensão manual, respectivamente, as estimativas de $\mathrm{h}^{2}$ foram de 10,18 e $19 \%$. Isto significa que há uma forte influência dos fatores ambientais não partilhados ou únicos de cada membro da fratria, associados a comportamentos de natureza cultural própria das famílias. Estes resultados são inferiores aos frequentemente reportados na literatura. Por exemplo, no estudo desenvolvido por KATZMARZYK et al. (2001) em 1.264 indivíduos canadenses aparentados de ambos os sexos dos sete aos 69 anos, os resultados de $\mathrm{h}^{2}$ foram de 37,59 e 48\% para os testes de flexão de braços, abdominais e preensão manual, respectivamente. Mais recentemente, em uma amostra com 41 pares 
de gêmeos monozigóticos e dizigóticos, THOMIS, VAN Leemputte, Maes, Blimkie, Claessens, Marchal, Willems, Vlietinck e Beunen (1997) referiram que os fatores genéticos explicaram entre $66-78 \%$ da variabilidade observada na força muscular. Em contra partida, no estudo de Bouchard, Malina e Pérusse (1997) e Pérusse, Leblanc e Bouchard (1988) em famílias nucleares, a estimativa de $\mathrm{h}^{2}$ para a preensão manual foi muito baixa, praticamente zero, tal como calculado em Calanga, ainda que só tenhamos utilizado irmandades. No estudo canadense, a dimensão amostral foi de 1.630, enquanto que em Calanga a amostra é relativamente reduzida, somente 330. É também possível que aspectos de natureza cultural possam condicionar os desempenhos pelo fato da estrutura dos movimentos dos testes ser inabitual.

O teste de vaivém $(10 \times 5 \mathrm{~m})$ é uma prova que pretende avaliar a agilidade. Da variância total do desempenho neste fenótipo, apenas 7\% é atribuída a fatores genéticos. Este resultado sugere uma forte influência ambiental. É difícil interpretar este resultado, dada a falta de estudos neste domínio com este fenótipo. Contudo, aspectos de natureza cultural e motivacional podem apresentar alguma influência no valor obtido (VARELA-SILVA, 1996).

A corrida da milha é um excelente marcador da aptidão aeróbia de crianças e jovens. A sua validade concorrente tem sido frequentemente salientada na literatura. A aptidão aeróbia é um fenótipo que tem sido objeto de forte análise e controvérsia interpretativa no que se refere à sua dependência genética. As estimativas de $\mathrm{h}^{2}$ mostram uma grande variabilidade, de zero a 90\%, dependendo do fenótipo utilizado, da dimensão amostral, do tipo de análise estatística, bem como da presença ou ausência de ajustamento para distintas covariáveis (Bouchard, Malina \& Pérusse, 1997; BOUCHARD et al., 1998, 1999; PÉrusse et al., 2001). As estimativas mais viáveis situam o valor $\mathrm{de}^{2} \mathrm{em}$ torno de 50\% (BOUCHARD \& RANKINEN, 2001). No HERITAGE Family Study é referida uma contribuição materna de $30 \%$ para a expressão deste fenótipo, possivelmente devido a transmissão mitocondrial (BOUCHARD, MALINA \& PérusSE, 1997). Na presente pesquisa, os valores são semelhantes às estimativas mais conservadoras para $\mathrm{o}$ consumo máximo de $\mathrm{O}_{2}$, ou seja, aproximadamente $49 \%$. É claro que os valores $\mathrm{h}^{2}$ observados no presente estudo sugerem uma forte influência genética.

Com o propósito de ajudar a interpretar os valores $\mathrm{de} \mathrm{h}^{2}$ estimados neste estudo, recorremos aos valores das correlaçôes das distintas fratrias. Nos diferentes pares de irmãos registramos resultados baixos a moderados $(-0,01 \leq \mathrm{r} \leq 0,45)$ em provas como o sentar e alcançar, preensão manual, abdominais, corrida vaivém e tempo de suspensão na barra, salientando a importância de fatores ambientais não partilhados ou únicos de cada membro da fratria. Em contrapartida, em outras provas entre fratrias do mesmo sexo e sexo distintos, a agregação é moderada. Diversos fatores podem ajudar a interpretar os resultados encontrados nas correlaçôes: 1) reduzida dimensão amostral, não obstante termos amostrado $\approx 10 \%$ da população escolar; este fato limita a potência dos testes estatísticos e induz a presença de um erropadrão relativamente grande não permitindo que as correlações sejam significativamente diferentes de zero; 2) aspectos de natureza socioeconômica e cultural e de relações interpessoais próprias desta população. Por exemplo, a baixa instrução das famílias, diferenças na dimensão do agregado familiar, problemas com cuidados primários de saúde, insuficiência nutricional, hierarquia na distribuição das tarefas de subsistência no seio das famílias em função do sexo e da idade dos sujeitos, como atividades agrícolas, pastorícia e atividades domésticas como cozinhar, cuidar das crianças e carregar água (BRUN, BLEIBERG \& Goihman, 1981; Giampietro \& Pimentel, 1992; Noronha, Alecrim, Adolfo, Romero \& Macedo, 2000; Prista, Marques \& Maia, 1997) podem condicionar os resultados da semelhança familiar. Por outro lado, fatores de natureza biológica como a incidência de doenças infectocontagiosas, doenças inflamatórias e perfil endêmico da malária da zona do estudo (AbBi, Christian, Gujaral \& Gopaldas, 1988; Brown \& Konner, 1987), parecem também interferir nos níveis de agregação observados. A estes aspectos adiciona-se a manifestação de diferenças sexuais nos valores da massa corporal resultantes das alterações morfológicas típicas deste intervalo etário, salientado maiores ganhos de massa muscular nos meninos e em maiores valores de gordura corporal nas meninas. Todos estes fatores podem condicionar de modo distinto o desempenho dos sujeitos nas diferentes componentes da aptidão física (MALINA \& BOUCHARD, 1991), sobretudo neste grupo de Calanga. Em geral, os resultados da presente pesquisa mostram que a variabilidade nas componentes da aptidão física das crianças e jovens de Calanga, é consequência de um forte efeito do ambiente não comumente partilhado, relacionado a um estilo de vida próprio daquela comunidade de subsistência, que origina rotinas do dia-a-dia distintos entre os membros das famílias nucleares. 


\section{Conclusões}

De acordo com os resultados encontrados conclui-se que no conjunto das sete componentes da aptidão física: 1) as irmãs evidenciaram maior semelhança entre si do que os irmãos, e irmãos de sexo oposto; 2) a relevância dos efeitos genéticos que governam diferenças interindividuais fez-se sentir na flexibilidade, na capacidade aeróbia e na força explosiva dos membros inferiores; 3) nos restantes fenótipos (força dos membros superiores, força de preensão manual, força/resistência abdominal e agilidade) os resultados sugerem que a maior porção da variabilidade pode ser explicada por fatores ambientais não transmissíveis dentro das famílias nucleares relacionados com atividades de subsistência familiar.

\section{Abstract}

Sibling clustering in physical fitness: a study in Calanga, a rural region of Mozambique.

This study has two aims: 1) to determine familial clustering in different physical fitness components and 2) to estimate its genetic component $\left(h^{2}\right)$. We sampled 330 subjects ( 174 males and 156 females) aged seven to 17 years, belonging to 132 families from Calanga, a rural locality in Mozambique. Physical fitness was assessed with the following tests: sit-and-reach (SR), standing long jump (SL), flexed arm hang (FHA), hand grip $(\mathrm{HG})$, 1-mile run (1M), curl-ups (CU), and shuttle run $10 \times 5$ meters (SHR). Correlations were used to estimate sibling clustering, and genetic factors were computed using a variance components approach within a maximum likelihood framework. All computations were adjusted for height, weight, age, sex, age ${ }^{2}$, age ${ }^{3}$, sex*age, sex*age ${ }^{2}$ as well as a physical activity score. SAGE, a statistical package for Genetic Epidemiology, was used in all analysis.Correlations within each fitness component were low: brother-brother (between -0.01 and 0.25 ), sister-sister (between -0.19 and 0.45 ) and brother-sister (between -0.02 and 0.39 ). The genetic component in each fitness component was: $S R h^{2}=0.41 \pm 0.16, p=0.01 ; A \sqcup h^{2}=0.60 \pm 0.14, p$ $=0.001 ; \mathrm{FHA} \mathrm{h}^{2}=0.18 \pm 0.20, p=0.37 ; \mathrm{CU} \mathrm{h}^{2}=0.09 \pm 0.13, p=0.59 ; \mathrm{HG} h^{2}=0.19 \pm 0.13, \mathrm{p}=0.16 ; \mathrm{SHR}$ $h^{2}=0.07 \pm 0.15, p=0.63 ; 1 \mathrm{M} \mathrm{h}^{2}=0.49 \pm 0.14, p=0.001$. Conclusions: 1 ) familial aggregation was higher between sisters than between brothers or siblings of opposite gender; 2 ) moderate genetic factors were found in flexibility, aerobic capacity and explosive strength; 3 ) variance between siblings in the other phenotypes were mostly explained by environmental factors that may be related to family subsistence activities.

UnIterms: Epidemiological genetics; Siblings; Physical fitness; Mozambique.

\section{Referências}

ABBI, R.; CHRISTIAN, P.; GUJARAL, S.; GOPALDAS, T. Mother's nutrition knowledge and child nutrition status in India. Food and Nutrition Bulletin, Tokyo, v.10, n.3, p.51-4, 1988.

AMERICAN ALLIANCE FOR HEALTH, PHYSICAL EDUCATION, RECREATION AND DANCE (AAHPERD). AAHPERD health related fitness test manual. Reston: AAHPERD, 1980.

BEUNEN, G.; THOMIS, M.A. Muscular strength development in children and adolescents. Pediatrics Exercise Sciences, Champaign, v.12, n.2, p.174-97, 2000.

BEUNEN, G.; THOMIS, M.; PEETERS, M.; MAES, H.H.; CLAESSENS, A.L.; VLIETINCK, R. Genetics of strength and power characteristics in children and adolescents. Pediatrics Exercise Sciences, Champaign, v.15, n.2, p.128-38, 2003. BOUCHARD, C.; AN, P.; RICE, T.; SKINNER, J.S.; WILMORE, J.H.; GAGNON, J.; PÉRUSSE, L.; LEON, A.S.; RAO, D.C. Familial aggregation of $\mathrm{VO}_{(2 \max )}$ response to exercise training: results from the HERITAGE Family Study. Journal of Applied Physiology, Berlin, v.87, n.3, p.1003-8, 1999.

BOUCHARD, C.; DAW, E.W.; RICE, T.; PERUSSE, L.; GAGNON, J.; PROVINCE, M.A.; LEON, A.S.; RAO, D.C.; SKINNER, J.S.; WILMORE, J.H. Familial resemblance for $\mathrm{VO}_{2 \max }$ in the sedentary state: the HERITAGE Familiy study. Medicine and Science in Sports and Exercise, Madison, v.30, n.2, p.252-8, 1998. 
BOUCHARD, C.; MALINA, R.M.; PÉRUSSE, L. Genetic of fitness and physical performance. Champaign: Human Kinetic, 1997. BOUCHARD, C.; RANKINEN, T. Individual differences in response to regular physical activity. Medicine and Science in Sports and Exercise, Madison, v.33, n.6, S446-51 (discussion S52-3), 2001. Supplement.

BROWN, P. J.; KONNER, M. An anthropological perspective on obesity. Annals of the New York Academy of Sciences, New York, v.499, p.29-46, 1987.

BROWNSON, R. C.; BOEHMER, T.K.; LUKE, D.A. Declining rates of physical activity in the United States: what are the contributors? Annual Review of Public Health, Palo Alto, v.26, p.421-43, 2005.

BRUN, T.; BLEIBERG, F.; GOIHMAN, S. Energy expenditure of male farmers in dry and rainy seasons in Upper-Volta. British Journal of Nutrition, Cambridge, v.45, n.1, p.67-75, 1981.

CASE WESTERN RESERVE UNIVERSITY. Department of Epidemiology and Biostatistics. SAGE: Statistical analysis for genetic epidemiology. Cleveland: Case Western Reserve University, 2005.

CORDAIN, L.; GOTSHALL, R.W.; EATON, S.B.; EATON, S.B. Physical activity, energy expenditure and fitness: an evolutionary perspective. International Journal of Sports Medicine, Stuttgart, v.19, n.5, p.328-35, 1998.

CORLET, J.T. Strength development of Tswana children. Human Biology, Detroit, v.60, n.4, p.569-77, 1988.

CONSEIL DE L'EUROPE. EUROFIT: tests européens d'aptitude physique. Strasbourg: Conseil de L'Europe,1993.

COUNCIL OF EUROPE. EUROFIT: Handbook for the EUROFIT tests of physical fitness. Rome: Council of Europe / Committee for the Development of Sport, 1988.

ELNASHIR, A.M.; MAYHEW, J.L. Physical fitness status of Egyptian children aged 9-18 years. British Journal of Sports Medicine, London, v.18, n.1, p.26-9, 1984.

FAYE, J.; SECK, K.; CISSE, F. Transverse study comparing certain physical characteristics of Senegalese children and adolescents from 7 to 13 years of age. Dakar Medical, Dakar, v.44, n.2, p.194-8, 1999.

FERNANDES, S.C.T.C.; MAIA, J.A.R. O código relacional da actividade física e aptidão física associada à saúde: efeito genéticos e ambientais. Porto: Faculdade do Desporto, Universidade do Porto, 2006.

GIAMPIETRO, M.; PIMENTEL, D. Energy efficiency and nutrition in societies based on human labour. Ecology of Food and Nutrition, New York, v.28. n.1-2, p.11-32, 1992.

GUESQUIÈRE, J.; D’HULST, C.; NKIAMA, E. Fitness and oxygen uptake of children in the Ituri forest: natural selection or adaptation to the environment? International Journal of Anthropology, Dordrecht, v.4, n.1-2, p.75-86, 1989.

HENNEBERG, M.; BRUSH, G.; HARRISON, G.A. Growth of specific muscle strength between 6 and 18 years in contrasting socioeconomic conditions. American Journal of Physical Anthropology, Hoboken, v.115, n.1, p.62-70, 2001.

KATZMARZYK, P.T.; GLEDHILL, N.; PÉRUSSE, L.; BOUCHARD, C. Familial aggregation of 7-year changes in musculoskeletal fitness. The Journals of Gerontology Series A: Biological Sciences and Medical Sciences, Washington, v.56, n.12, p.B497-502, 2001.

LOHMAN, T.G.; ROCHE, A.F.; MALINA, R.M. Anthropometric standardization reference manual. Champaign: Human Kinetics, 1988.

MAIA, J.; LOPES, V.; SEABRA, A.; GARGANTA, R. Efeitos genéticos e do envolvimento nos níveis de actividade física e aptidão física associada à saúde: Um estudo em gémeos dos 6 aos 12 anos de idade do Arquipélago dos Açores, Portugal.

Revista Brasileira de Ciência e Movimento, São Caetano do Sul, v.11, n.4, p.33-44, 2003.

MAIA, J.A.R.; LOPES, V.P.; MORAIS, F.P. Actividade física e aptidão física associada à saúde: um estudo de epidemiologia genética em gémeos e suas famílias realizado no arquipélago dos Açores. Porto: Faculdade de Ciências do Desporto e de Educação Física da Universidade do Porto/Direcção Regional de Educação Física e Desporto da Região Autónoma dos Açores, 2001. MALINA, R.; BOUCHARD, C. Growth maturation and physical activity. Champaign: Human Kinetics, 1991.

MALINA, R.M. Genetics of motor development and performance: sport and human genetics. Champaign: Human Kinetics, 1986. MOÇAMBIQUE. Instituto Nacional de Estátistica (INE). Recenciamento geral da população. Maputo: INE, 2006.

MURIA, A.; PRISTA, A.; MAIA, J. Estudo da validade das medidas critério do Fitnessgram para a população escolar de Maputo. In: PRISTA, A.; MARQUES, A.; MAIA, J. 10 anos de actividade científica. Lisboa: Centro de Estudos e Formação Desportiva; 2000. p.94-101. NKIAMA, E. Physical fitness status of school children et Bunia in Zaire: world wide variation in physical fitness. Leuven: Katholieke Universiteit Leuven, 1993. p.126-30.

NORONHA, E.; ALECRIM, M.G.C.; ADOLFO, A.; ROMERO, S.; MACÊDO, M. Estudo clínico da malária falciparum em crianças em Manaus, AM, Brasil. Revista da Sociedade Brasileira de Medicina Tropical, Uberaba, v.33, n.2, p.185-90, 2000. PÉRUSSE, L.; GAGNON, J.; PROVINCE, M.A.; RAO, D.C.; WILMORE, J.H.; LEON, A.S.; BOUCHARD, C.; SKINNER, J.S. Familial aggregation of submaximal aerobic performance in the HERITAGE Family study. Medicine and Science in Sports and Exercise, Madison, v.33, n.4, p.597-604, 2001. 
PÉRUSSE, L.; LEBLANC, C.; BOUCHARD, C. Inter-generation transmission of physical fitness in the Canadian population. Canadian Journal of Sport Science, Champaign, v.13, n.1, p.8-14, 1988.

PÉRUSSE, L.; LEBLANC, C.; TREMBLAY, A.; ALLARD, C.; THERIAULT, G.; LANDRY, F.; TALBOT, J.; BOUCHARD, C. Familial aggregation in physical fitness, coronary heart disease risk factors, and pulmonary function measurements. Preventive Medicine, Baltimore, v.16, n.5, p.607-15, 1987a.

PÉRUSSE, L.; LORTIE, G.; LEBLANC, C.; TREMBLAY, A.; THERIAULT, G.; BOUCHARD, C. Genetic and environmental sources of variation in physical fitness. Annals of Human Biology, London, v.14, n.5, p.425-34, 1987b.

PRISTA, A. Crescimento, actividade física e aptidão física em países não industrializados: abordagem biocultural em crianças e jovens de Moçambique. Revista Agon, Porto, v.2, p.85-102, 1995.

PRISTA, A.; MARQUES, A.T.; MAIA, J.A. Empirical validation of an instrument to measure habitual physical activity in youth from Maputo, Mozambique. American Journal of Human Biology, New York, v.12, n.4, p.437-46, 2000.

PRISTA, A.; MARQUES, A.T.; MAIA, J.A.R. Relationship between physical activity, socioeconomic status and physical fitness of 8-15 year old youth from Mozambique. American Journal of Human Biology, New York, v.9, n.4, p.449-57, 1997.

SALLIS, J.F.; PATTERSON, T.L.; MORRIS, J.A.; NADER, P.R.; BUONO, M.J. Familial aggregation of aerobic power: the influence of age, physical activity, and body mass index. Research Quarterly for Exercise and Sport, Washington, v.60, n.4, p.318-24, 1989.

SARANGA, S.; PRISTA, A.; MAIA, J. Mundanças nos níveis de aptidão física em função de alterações políticas e sócioeconómicas de 1992-1999. In: PRISTA, A.; MAIA, J.; SARANGA, S.; MARQUES, A. Saúde, crescimento e desenvolvimento: um estudo epidemiológico em crianças e jovens de Moçambique. Porto: FADE-UP, 2002. p. 71-88.

SHEPHARD, R.J. Aging, physical activity and health. Toronto: Human Kinetics, 1997.

SILBERG J.R.M.; MEYER, J.; MAES, H.; HEWITT, J.; SIMONOFF, E.; PICKLES, A.; LOEBER, R.; EAVES, L. Genetic and environmental influences on the covariation between hyperactivity and conduct disturbance in juvenile twins. Journal of Child Psychology and Psychiatry, London, v.37, n.7, p.803-16, 1996.

THOMIS, M. A.; VAN LEEMPUTTE, M.; MAES, H.H.; BLIMKIE, C.J.; CLAESSENS, A.L.; MARCHAL, G.; WILLEMS, E.; VLIETINCK, R.F.; BEUNEN, G.P. Multivariate genetic analysis of maximal isometric muscle force at different elbow angles. Journal of Applied Physiology, Berlin, v.82, n.3, p.959-67, 1997.

VARELA-SILVA, M.I. Influência de variáveis bioculturais na motivação para a participação desportiva. 1996. Tese (Mestrado) Faculdade de Motricidade Humana, Universidade Técnica de Lisboa, Lisboa, 1996.

VASQUES, C.M.S. Semelhanças familiares na actividade física, aptidão física e somatótipo: um estudo em famílias nucleares e em pares de irmãos. Porto. Dissertação (Mestrado em Desporto para Crianças e Jovens) - Faculdade do Desporto, Universidade do Porto, Porto, 2005.

WIGGINTON, J.E.; ABECASIS, G.R. PEDSTATS: descriptive statistics, graphics and quality assessment for gene mapping data. Bioinformatics, Oxford, v.21, n.16, p.3445-7, 2005.

ENDEREÇO

José António Ribeiro Maia Laboratório de Cineantropometria Faculdade de Desporto Universidade do Porto R. Dr. Plácido Costa, 91 4200-450 - Porto - PORTUGAL e-mail: jmaia@fade.up.pt
Recebido para publicação: 04/03/2009

Revisado em: 18/01/2010

Aceito: 18/02/2010 